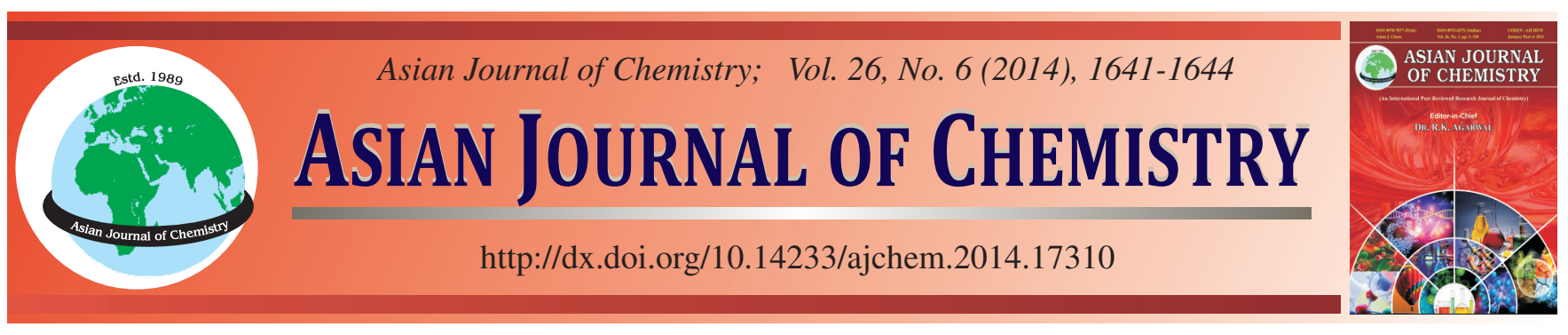

\title{
Electro-Optical Property of Plasma Enhanced Chemical Vapor Deposition ZnO:Al Films Influenced by Substrate Materials and Annealing Treatments $\dagger$
}

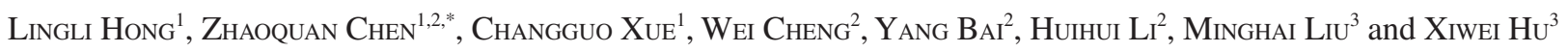

${ }^{1}$ College of Materials Science \& Engineering, Anhui University of Science and Technology, Huainan 232001, P.R. China ${ }^{2}$ College of Electrical \& Information Engineering, Anhui University of Science and Technology, Huainan 232001, P.R. China ${ }^{3}$ State Key Laboratory of Advanced Electromagnetic Engineering and Technology, Huazhong University of Science and Technology, Wuhan 430074, P.R. China

*Corresponding author: E-mail: zqchen@aust.edu.cn

In this paper, $\mathrm{AZO}(\mathrm{ZnO}: \mathrm{Al})$ polycrystalline thin films with strong adhesion to the substrate, as low as $89 \Omega / \%$ of square resistance and as high as $85 \%$ of visible light transmittance, have been fabricated by PECVD (plasma enhanced chemical vapor deposition) method depositing on glass substrate and silicon substrate, respectively. Based on PECVD method, the electro-optical property of the proposed AZO films influenced by substrate materials and annealing treatments is studied in detail. the fabrication process of AZO film is also shown. The results suggested that AZO film fabricated by PECVD is a meaningful attempt for the industrial applications.

Keywords: AZO (ZnO:Al), PECVD, Transparent conductive film, Electro-optical property.

\section{INTRODUCTION}

The plasmas having active atoms or atomic groups are attributed for the production of some special materials. Plasma enhanced chemical vapor deposition (PECVD) method is to deposit the compound film on the low-temperature substrate in plasmas yielded by gaseous discharges. When applies the high electric voltage to the vessel with low gas pressure, few electrons existing in the gas will be easily accelerated to high speed by the electric field, due to its longer mean free path and higher speed electrons losing its energy by colliding with the neutral atom or the molecule. These particles are chemically active, may produce new particles and finally can participate in chemical reaction. The new produced electrons continually are accelerated and collided, which ionize the gas rapidly and form the plasmas eventually. Under the plasmas with the condition of low temperature, the film will grow up on the nonthermally substrate with fewer damage and reaction. Because of these processing merits, we may expect that PECVD method in the micro electron industrial application has a good prospect in future ${ }^{1-3}$.

The transparent conductive film is one kind of film material, which have the advanced performances with electric conduction and optic transmittance in the scope of visible light. Metal like $\mathrm{Au}, \mathrm{Ag}, \mathrm{Pt}, \mathrm{Cu}, \mathrm{Rh}, \mathrm{Pd}, \mathrm{Al}, \mathrm{Cr}$, etc. when forms the thin film with the thickness from 3 to $15 \mathrm{~nm}$, also has visible light permeation in some degree and is applied as the transparent electrode in history ${ }^{4}$. However, due to the metallic film too strong to light absorption, some degree of weak hardness and its bad stability, hence the transparent conductive films, like oxide, nitride, fluoride are produced to instead of the metallic film. Recently, the transparent conducting oxides (i.e., TCO) constituted by metal oxides have already occupied the dominant position in its application domain, even this industrial demands have expanded unceasingly in recent years.

Therefore, the research on AZO ( $\mathrm{ZnO}: \mathrm{Al})$ film has become hotly in TCO domain recently. For example, the fabrication of the magnetron sputtering method has its merits of large area preparation with high doping rate $^{1}$, which is still the most widespread preparation technology for AZO film. The process based on sol-gel law does not need the vacuum condition, then many researchers ${ }^{5,6}$ have prepared AZO film with this method. The pulsed laser sedimentation may accurately control the quantity of the chemistry ingredient, which is easy to realize the multilayered film growth. There are many others methods, namely, vacuum evaporation, chemical vapour deposition and

Fresented at The 7th International Conference on Multi-functional Materials and Applications, held on 22-24 November 2013, Anhui University of Science \& Technology, Huainan, Anhui Province, P.R. China 
others ${ }^{4}$, which has been used to produce AZO film differently. In our previous report ${ }^{7}$, we had already carried on the research about the film electro-optic performance, the film crystal structure, the film growth factor and the crystal ingredient. Here, we will further discuss the electro-optical property of the proposed AZO films influenced by substrate materials and annealing treatments.

\section{RESULTS AND DISCUSSION}

Influence for the film surface appearance: Fig. 1(a, b) are the AZO film surface appearance image depositing on glass substrate and $\mathrm{Si}$ substrate. As shown in graph, we can found that the AZO film appearance on the two diffenrent kinds of substrate is similar and the difference of the crystal grain is little. AZO film on Si substrate is more compact and evener and its surface is smoother, but the film on glass substrate appears the flaw. This indicated that polishing the surface of the $\mathrm{Si}$ unit crystal is to be more advantageous in the AZO film growth compared with the amorphous state of the glass surface. Therefore, the glass substrate with smooth surface and fewer impurities should be used.
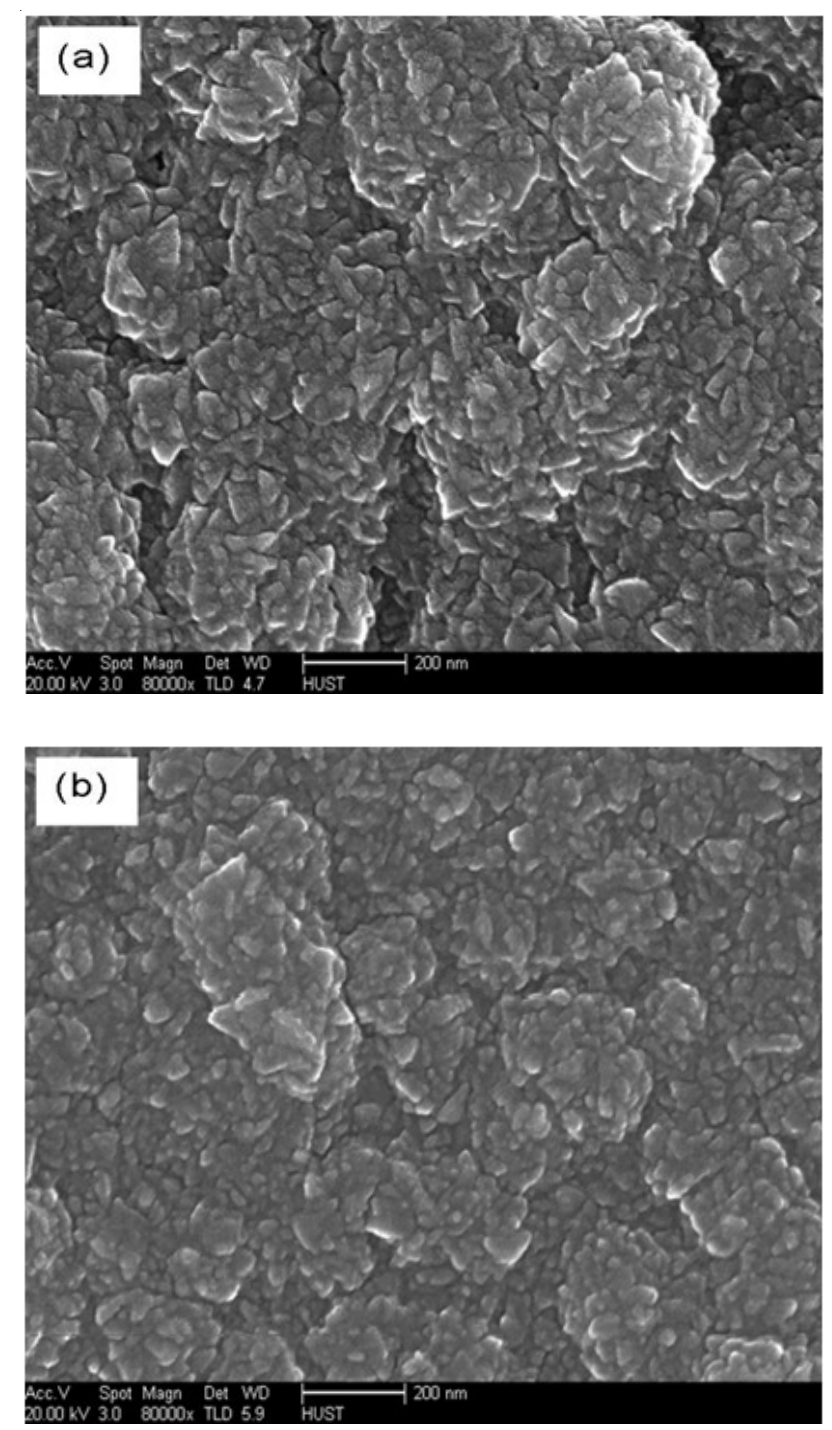

Fig. 1. FSEM image amplified by 80000 times: (a) glass substrate and (b) Si substrate
Influence for the film crystal structure: Compared with the XRD diagram of the AZO film on glass and Si substrate shown in Fig. 2(a, b), respectively. Although the structure of the substrate material is different, the structure of the AZO film is actually very close, the only difference is that the diffraction peak of the AZO film deposited on the glass substrate is intricate and which indicated that the monocrystalline silicon favours the AZO film growth in preferential crystal face.
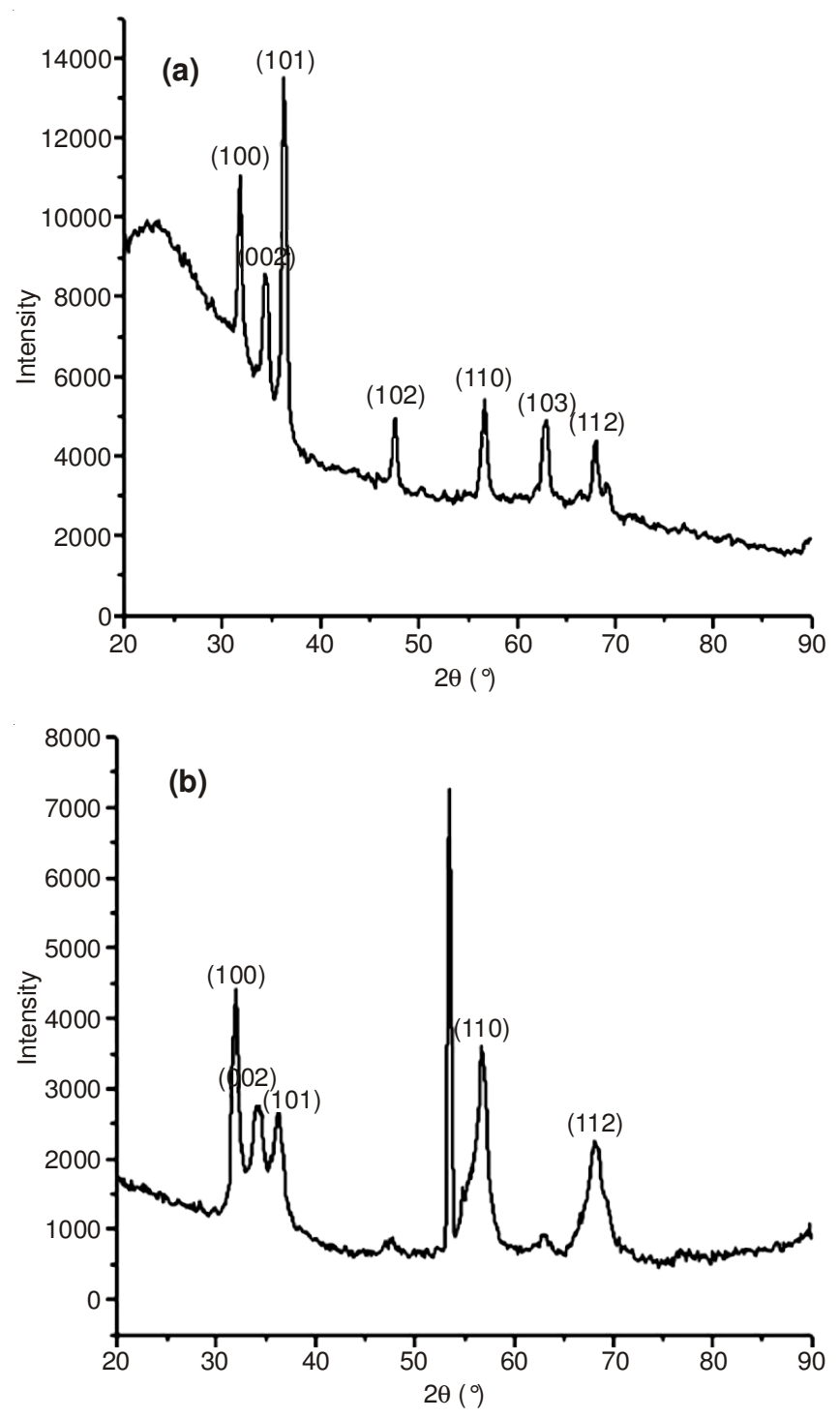

Fig. 2. XRD diagram: (a) glass substrate and (b) Si substrate

Influence of the doping quantity for the conductivity: The AZO film has two kinds of electric conduction mechanisms, namely the intrinsic donor and the external donor. The intrinsic donors present the $\mathrm{Zn}$ atom gap and the $\mathrm{O}$ vacancy and the external donor is $\mathrm{Al}$ ion displacement, as shown in following:

$$
\begin{aligned}
& \mathrm{ZnO}+\mathrm{xAl}^{3+} \rightarrow \mathrm{Zn}_{1-\mathrm{x}}^{2+}\left(\mathrm{Al}^{3+} \mathrm{e}\right)_{\mathrm{x}}+\mathrm{xZn}^{2+} \\
& \mathrm{ZnO} \rightarrow \mathrm{Zn}_{1-\mathrm{x}}^{2+}\left(\mathrm{Zn}^{2+} .2 \mathrm{e}\right)_{\mathrm{x}} \mathrm{O}_{1-\mathrm{x}}^{2-}+\frac{\mathrm{x}}{2} \mathrm{O}_{2} \uparrow
\end{aligned}
$$

Namely, under the perfect condition, each $\mathrm{Al}$ ion displacement provides one conduction electron and each $\mathrm{O}$ vacancy 
provides two conduction electrons. When the doping quantity increases from 1 to $3 \%$, the film resistivity reduces largely with lowest square electronic resistivity $89 \Omega / \%^{7}$.

Influence for the film crystal structure: Fig. 3(a, b) are the XRD diagram of the AZO film under the deposition temperature $200^{\circ} \mathrm{C}$ for annealing before and after, respectively. After annealing $1 \mathrm{~h}$ in air with $500^{\circ} \mathrm{C}$, the characteristics peak of the AZO film becomes remarkable and the half peak width (002) with $0.6298^{\circ},(110)$ with $0.4723^{\circ}$ and (100) with $0.4723^{\circ}$ are smaller than before annealing. It is obviously that after annealing the AZO film crystallization improves under the low temperature depositing.
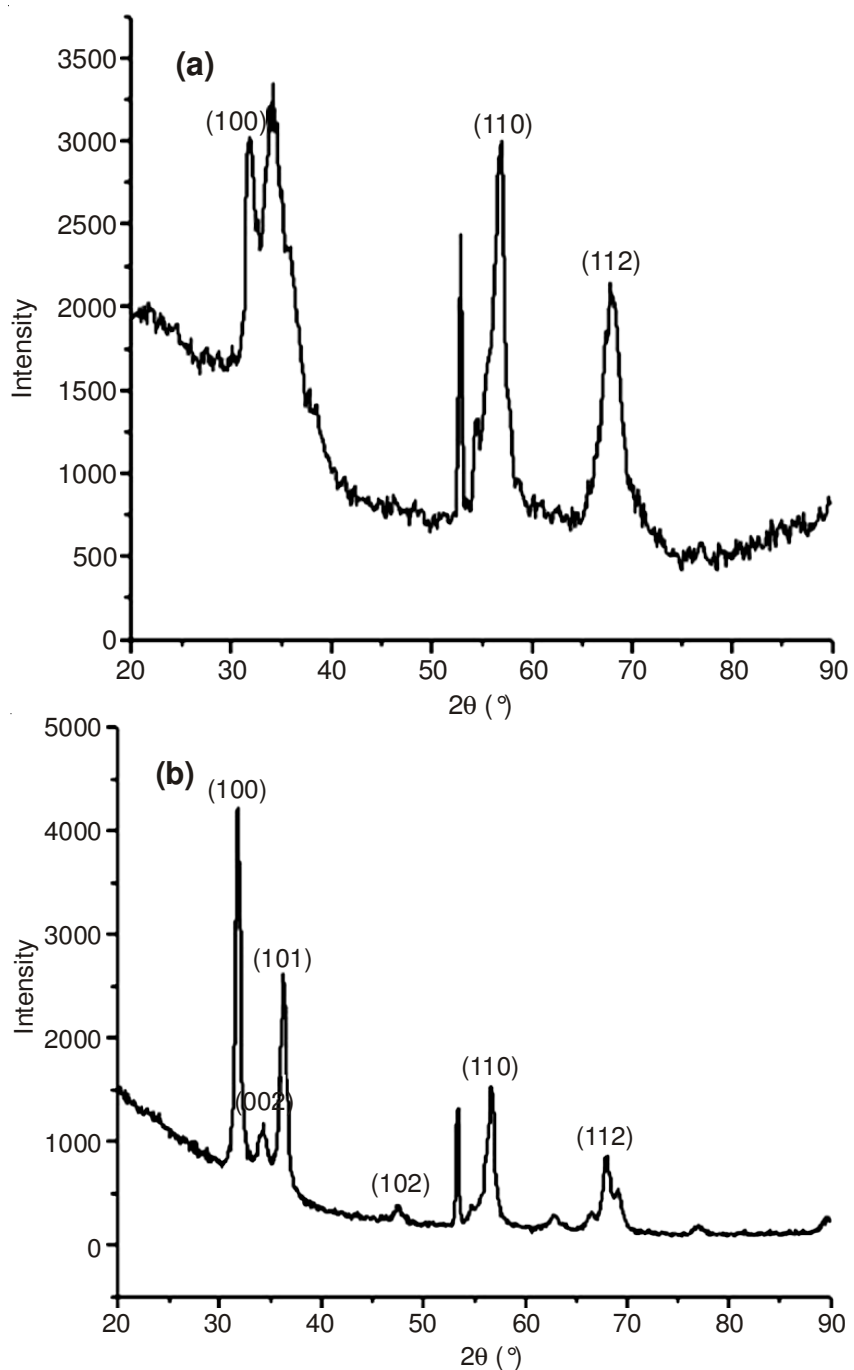

Fig. 3. XRD diagram: (a) before annealing and (b) after annealing

Fig. 4(a, b) are the XRD diagram of the AZO film under the deposition temperature $400^{\circ} \mathrm{C}$ for annealing before and after, respectively. After annealing $1 \mathrm{~h}$ in air with $500^{\circ} \mathrm{C}$, the characteristics peak of the AZO film also becomes remarkable but the change of crystal structure is very weak. That may be because the deposition temperature with $400^{\circ} \mathrm{C}$ is close to the annealing temperature with $500^{\circ} \mathrm{C}$.

Influence for the film optics performance: As shown in Fig. 5, the film optical transmissibility elevates to $85 \%$ after annealing with $500^{\circ} \mathrm{C}$ in atmosphere for $1 \mathrm{~h}$. It is because that the change of the film crystal structure causes the corresponding
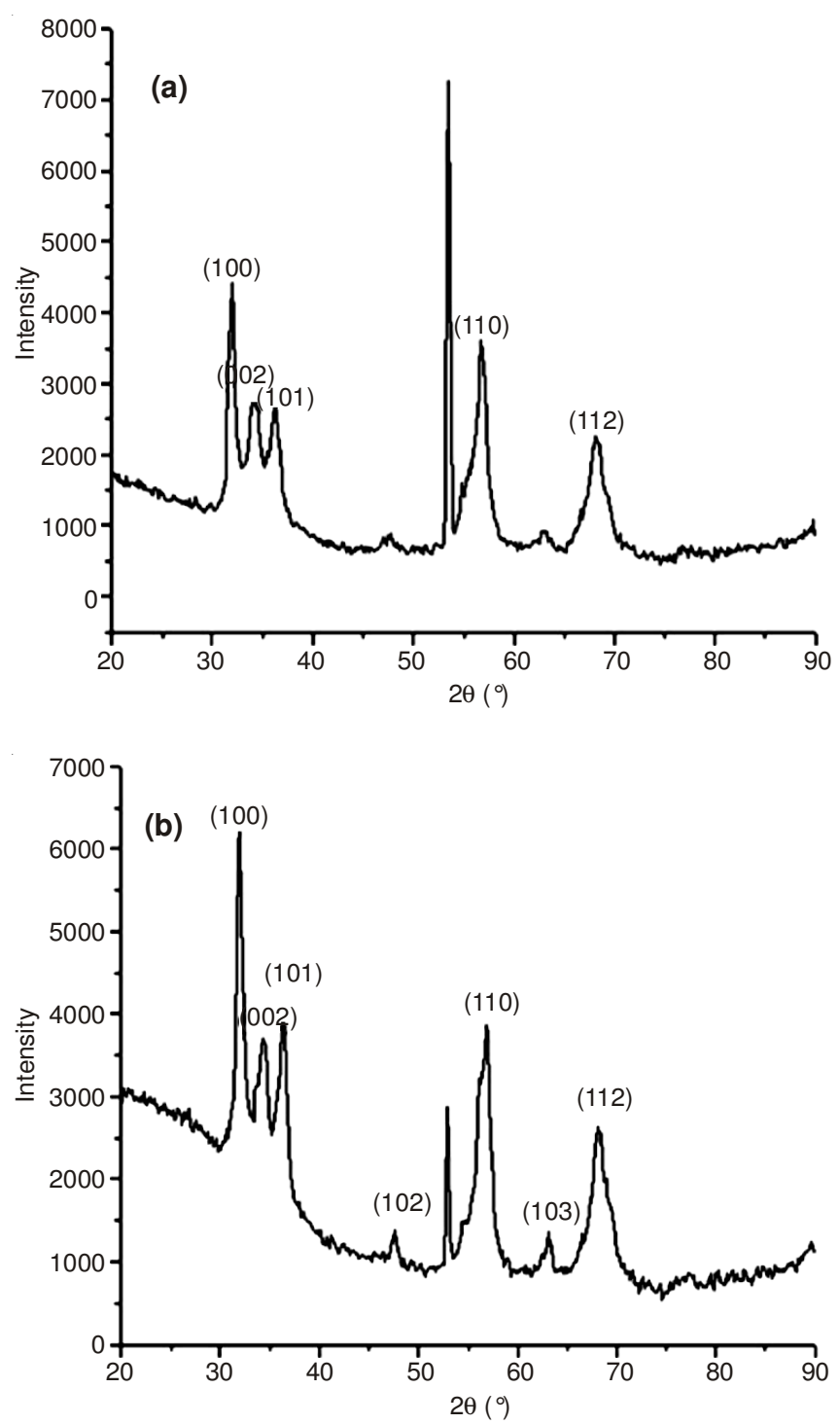

Fig. 4. XRD diagram: (a) before annealing and (b) after annealing

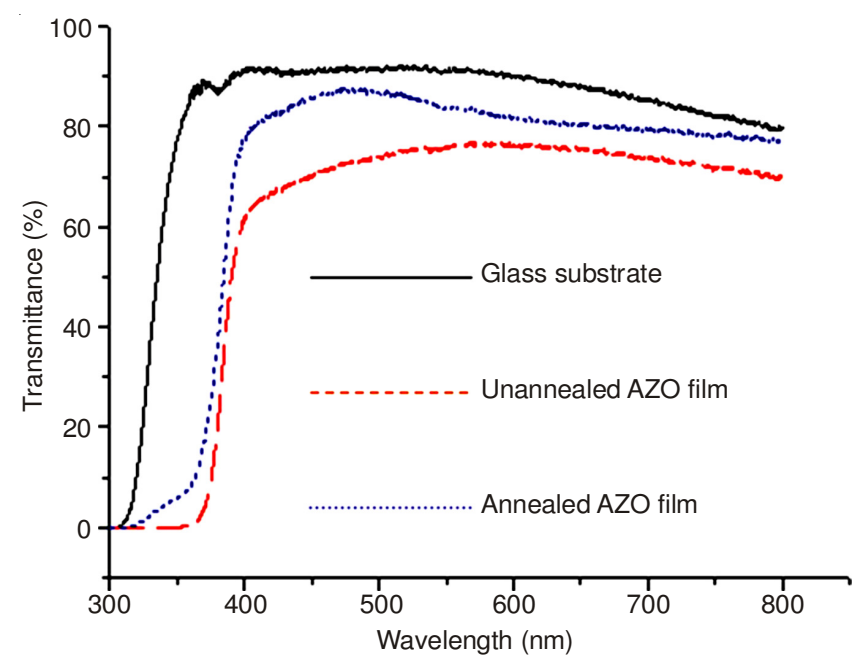

Fig. 5. Transmissivity diagram under the annealing temperature with 500 ${ }^{\circ} \mathrm{C}$ in air

change of the optical band gap. Unfortunately, we also found the film square resistance increasing synchronously. That may be because the increasing oxygen contents in the AZO film 
causes the density of the current carrier reducing after annealing in air. Aghamalyan et al $^{8}$ have found that after annealing in vacuum the light transmissibility of the AZO film enhances and the square resistance reduces.

\section{Conclusion}

We have carried out the experiments for examining the influence of the performance of AZO film like affecting factor, namely, the substrate material and the annealing treatment. The $\mathrm{ZnO}$ film with some degree of $\mathrm{Al}$ doping have caused film performance changes in the electricity, optics, structure and surface appearance. The doping quantity obviously affects the film electricity performance: (1) the AZO film has its lowest square resistance at the doping quantity 5 at \%; (2) depositing time with $0.5 \mathrm{~h}$, the film thickness is $1060 \mathrm{~nm}$ and the film simultaneously has the lowest square resistance $(89 \Omega / \%)$ and the high average transmittance $(85 \%)$ in the visible light scope.

\section{ACKNOWLEDGEMENTS}

This work is supported by the Natural Science Foundation of China (Grant No. 11105002), Open-end Fund of State Key
Laboratory of Structural Analysis for Industrial Equipment (Grant No. GZ1215), Natural Science Foundation for University in Anhui Province of China (Grant No. KJ2013A106) and Doctoral Scientific Research Funds of AUST.

\section{REFERENCES}

1. J.F. Chang, H.L. Wang and M.H. Hon, J. Cryst. Growth, 211, 93 (2000).

2. R.J. Hong, X. Jiang, B. Szyszka, V. Sittinger and A. Pflug, Appl. Surf. Sci., 207, 341 (2003).

3. W.W. Wang, X.G. Diao, Z. Wang, M. Yang, T.M. Wang and Z. Wu, Thin Solid Films, 491, 54 (2005).

4. A. Stadler, Materials, 5, 661 (2012).

5. R. Maity, S. Kundoo and K.K. Chattopadhyay, Sol. Energy Mater. Sol. Cells, 86, 217 (2005).

6. G.G. Valle, P. Hammer, S.H. Pulcinelli and C.V. Santilli, J. Eur. Ceram. Soc., 24, 1009 (2004)

7. Z.Q. Chen, L.J. Zhu, L.L. Hong, C.G. Xue, M.H. Liu, X.L. Zheng, Y.L. Hu, Q.B. Ye and X.W. Hu, Asian J. Chem., (Submitted).

8. N.R. Aghamalyan, I.A. Gambaryan, E.K. Goulanian, R.K. Hovsepyan, R.B. Kostanyan, S.I. Petrosyan, E.S. Vardanyan and A.F. Zerrouk, Semicond. Sci. Technol., 18, 525 (2003). 